\title{
Mitral valve repair for endocarditis can be performed 3 days after repair of a bleeding mycotic brain aneurysm
}

\author{
Ryushi Maruyama, MD, PhD, ${ }^{\text {a }}$ Akira Yamada, $\mathrm{MD}, \mathrm{PhD},{ }^{\mathrm{a}}$ Taku Sugiyama, $\mathrm{MD}, \mathrm{PhD},{ }^{\mathrm{b}}$ \\ Kosuke Ujihira, MD, ${ }^{\mathrm{a}}$ Naritomo Nishioka, MD, ${ }^{\mathrm{a}}$ Yutaka Iba, MD, PhD, ${ }^{\mathrm{a}}$ Eiichiro Hatta, MD, PhD, ${ }^{\mathrm{a}}$ \\ Yoshihiko Kurimoto, MD, PhD, ${ }^{\mathrm{a}}$ Katsuyuki Asaoka, $\mathrm{MD}, \mathrm{PhD},{ }^{\mathrm{b}}$ Katsuhiko Nakanishi, MD, PhD, ${ }^{\mathrm{a}}$ and \\ Keisuke Sakai, MD, $\mathrm{PhD},{ }^{\text {a }}$ Sapporo, Japan
}

\footnotetext{
From the Departments of acCardiovascular Surgery, and ${ }^{\mathrm{b}}$ Neurosurgery, Teine Keijinkai Hospital, Sapporo, Hokkaido, Japan.

Disclosures: Authors have nothing to disclose with regard to commercial support.

Received for publication April 17, 2015; revisions received Sept 18, 2015; accepted for publication Oct 6, 2015 available ahead of print Nov 21, 2015

Address for reprints: Ryushi Maruyama, MD, PhD, Department of Cardiovascular Surgery, Teine Keijinkai Hospital, 12-1-40, Maeda 1-jou, Teine-ku, Sapporo, Hokkaido, Japan 006-8555 (E-mail: ryushim@ hotmail.com). J Thorac Cardiovasc Surg 2016;151:e59-61 $0022-5223 / \$ 36.00$

Copyright (C) 2016 by The American Association for Thoracic Surgery http://dx.doi.org/10.1016/j.jtcvs.2015.10.026
}

Cerebrovascular complications occur in $10 \%$ to $40 \%$ of infective endocarditis (IE) patients. ${ }^{1}$ The optimal timing of surgical intervention for active IE with cerebrovascular complications has been controversial because the risk of perioperative intracranial hemorrhage remains unclear. We report a case of active IE in which early open repair was performed successfully in a situation in which cardiac surgeons and neurosurgeons collaborated closely.

\section{CASE REPORT}

A 26-year-old man was referred to our hospital because he had persistent high fevers of unclear etiology. At admission, his temperature was $40.0^{\circ} \mathrm{C}$, his blood pressure was $116 / 40 \mathrm{~mm} \mathrm{Hg}$, and his heart rate was $126 \mathrm{bpm}$. Blood tests revealed a white blood cell count of 17,050 per $\mu \mathrm{L}$, and an elevated $C$ reactive protein level of $18.07 \mathrm{mg} / \mathrm{dL}$. Ceftriaxone IV was started after Haemophilus parainfluenzae grew in blood cultures. Computed tomography (CT) scans showed splenic infarctions, right renal infarctions, and cerebral hemorrhagic infarctions on the right temporal lobe and left occipital lobe. Brain magnetic resonance imaging (MRI) confirmed the findings of the CT, and showed multiple "black dots" on the T2*-weighted sequence. These black dots suggest thrombosed mycotic aneurysms. Transthoracic and transesophageal echocardiograms revealed moderate mitral valve regurgitation, and large vegetations on both the anterior and posterior mitral leaflets, with diameters of 12 and $8 \mathrm{~mm}$, respectively (Figure 1 ).

The management of this case was discussed extensively in a collaborative effort among members of the cardiac and neurosurgery teams, because the patient had both mobile, large vegetations on the mitral valve, and brain hemorrhagic infarctions. The patient's hemodynamic status was stable, and despite the concerning imaging, no significant neurologic deficits were present. Therefore, the teams decided

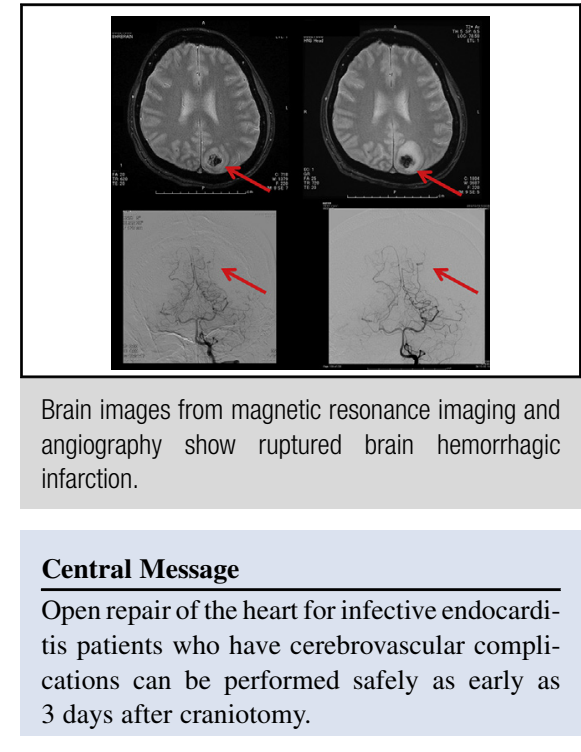

See Editorial Commentaries page e63 and e64.

to treat with antibiotic therapy for 1 week and then make a final decision. A week later, however, a brain CT scan followed by an MRI revealed enlargement of the cerebral hemorrhage in the left occipital lobe. The black dots found on the previous T2*-weighted brain MRI had not changed in shape or diameter. A brain angiogram revealed that the left posterior cerebral artery aneurysm had ruptured (Figure 2).

The teams decided to perform neurosurgery to resect the aneurysm, as a means to minimize the risk of cerebral hemorrhage enlargement during cardiopulmonary bypass, then follow with the cardiac operation as soon as possible. Neurosurgery was performed the next day, which consisted of open craniotomy with trapping of the mycotic aneurysmal feeding artery and resection of the mycotic aneurysm. No postoperative neurologic deficits were observed, and an MRI performed 2 days after the craniotomy confirmed that no hemorrhagic complication had occurred. Three days after the craniotomy, we performed the mitral valve repair. For cardiopulmonary bypass, the regular dose of heparin was administered, based on the postoperative MRI result.

We found a large vegetation ( $15 \mathrm{~mm}$ in diameter) and torn chordae on the A2 of the anterior mitral leaflet. Other vegetations ( $5 \mathrm{~mm}$ in diameter) were found on P1 and P3 of the 


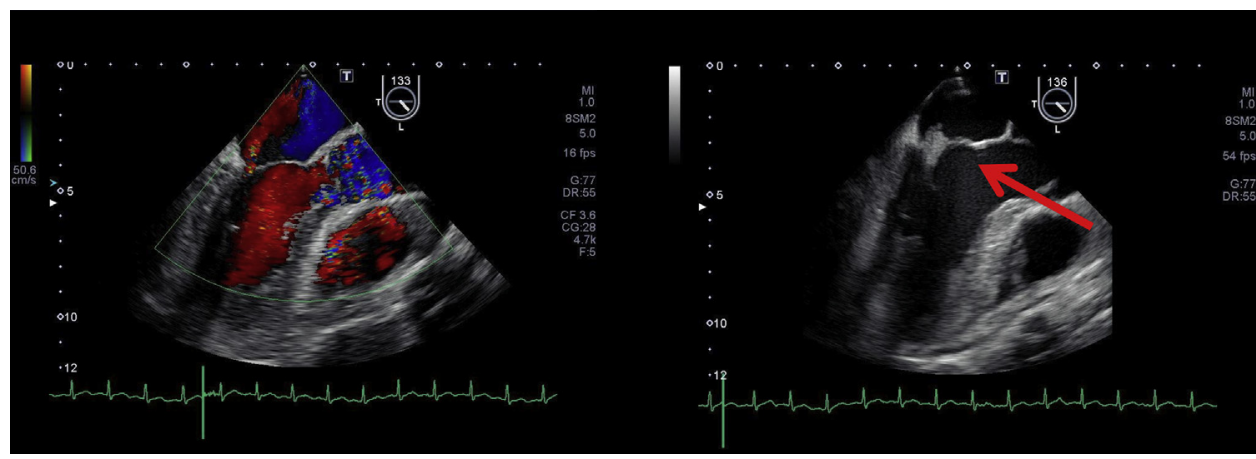

FIGURE 1. Transesophageal echocardiography showed moderate mitral valve regurgitation and vegetations on both the anterior mitral leaflet and posterior mitral leaflet (arrows).

posterior mitral leaflets. Even though the vegetations were friable and mobile, no annular abscess was found. The vegetations on both mitral leaflets were carefully resected. The mitral valve repair consisted of reconstruction with artificial chordae (2 pairs) for A2, using the loop technique with CV5 , triangular resection and suture of $\mathrm{P} 3$, posterior mitral leaflet cusp augmentation using glutaraldehyde-treated autopericardium, and finally, ring annuloplasty using a 32-mm Carpentier-Edwards Physio ring (Edwards Lifesciences, Irvine, Calif).

A brain CT scan done immediately after the operation found no hemorrhage. Postoperative transthoracic echocardiography revealed no mitral valve regurgitation, nor recurrence of IE. No neurologic deficits were identified in the following days, and after 6 weeks of antibiotic treatment, the patient was discharged in excellent condition.

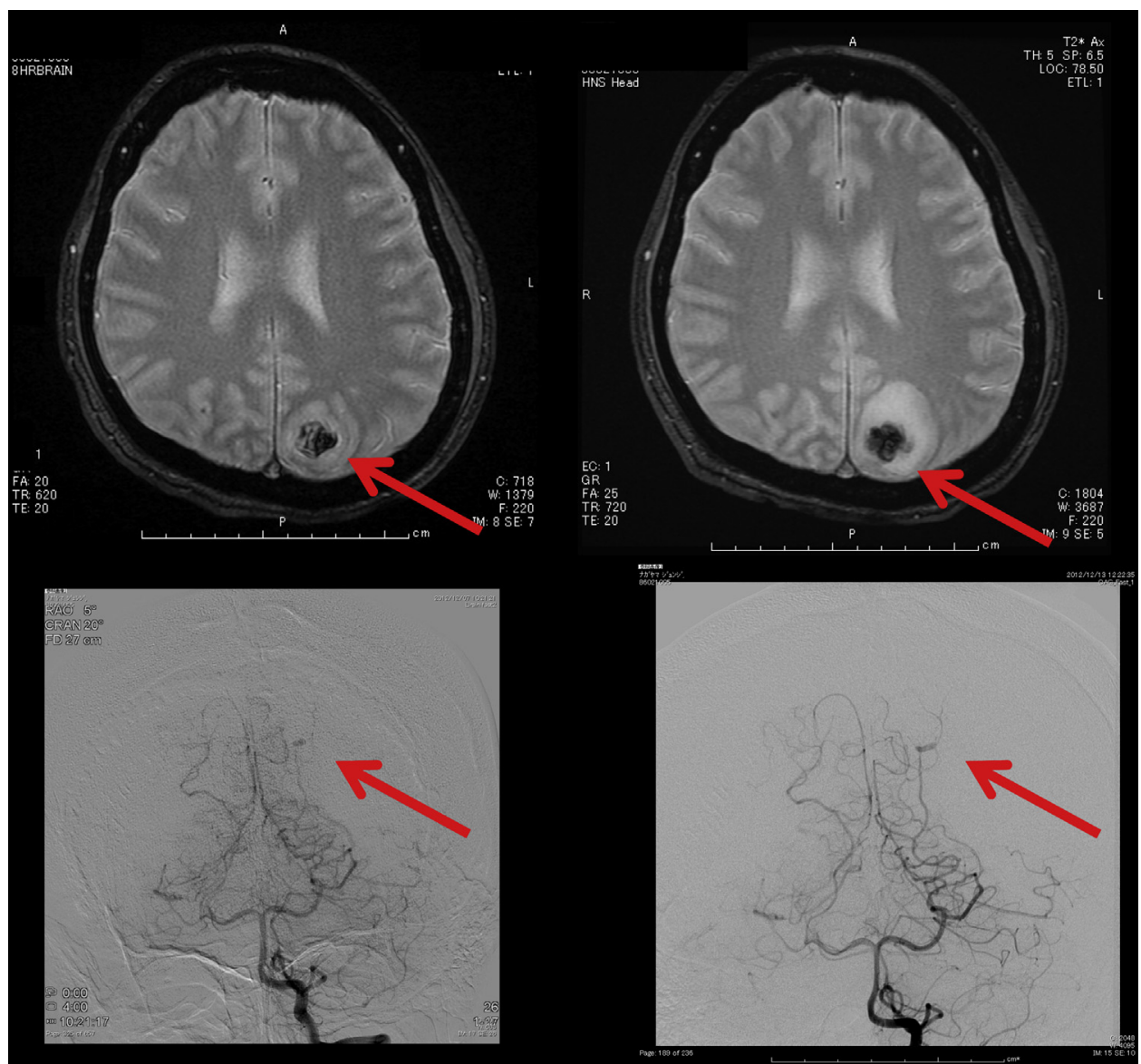

FIGURE 2. Magnetic resonance imaging showed enlargement of the cerebral hemorrhagic infarction in the left occipital lobe. Brain angiography revealed a ruptured left posterior cerebral arterial aneurysm (arrows). 


\section{DISCUSSION}

One of the most severe complications of IE is mycotic intracranial aneurysm, which may result in hemorrhagic brain infarctions. The optimal timing of surgical intervention for active IE with cerebrovascular complications has been controversial, because the risk of perioperative intracranial hemorrhage is unclear. The dilemma is that of which operation should be performed first: cardiac surgery or neurosurgery. Heparinization and hypotension during cardiopulmonary bypass could increase cerebral damage, such as hemorrhage, ischemic injury, or edema. If the craniotomy is done first, a cardiac complication or another brain stroke may occur.

Kin and colleagues ${ }^{2}$ showed that in IE patients, black dots on T2*-weighted brain MRI are a feature of thrombosed mycotic aneurysms and are thought to be secondary to hemosiderin deposition after a microbleed. These black dots reflect a high risk for cerebral hemorrhage. In our case, in addition to the ruptured mycotic aneurysm, other, multiple black dots were visible; however, because of their locations, neurosurgery to resect them was not considered feasible.

During the cardiopulmonary bypass surgery, we administered the regular dose of heparin, based on the postoperative MRI and the satisfactory healing of the craniotomy incision. We did not use nafamostat mesilate to reduce the heparin dose. Recently, Sakamoto and colleagues ${ }^{3}$ reported the efficacy of nafamostat mesilate during cardiopulmonary bypass for early surgery in patients who have active IE complicated by stroke. In retrospect, we believe this option might have been appropriate. Fortunately, in our case, no brain hemorrhage occurred after the cardiac surgery.

Asai and colleagues ${ }^{4}$ reported a case in which the cardiac operation was performed a day after the brain endovascular intervention. In our case, the lesion was too distal for an endovascular approach. Therefore, to trap and resect the brain hemorrhagic aneurysm, craniotomy was chosen as the appropriate approach.

In a study evaluating the timing of surgery in patients with IE and cerebral complications, Gillinov and colleagues $^{5}$ suggested that the interval between craniotomy and cardiac surgery should be 2 to 3 weeks. Fukuda and colleagues ${ }^{6}$ reported the mean interval between surgeries to be $26.7 \pm 21.8$ days. Case reports from Fujii and colleagues $^{7}$ and Kuki and colleagues ${ }^{8}$ found intervals of 3 and 8 days, respectively. The clinical practice guideline of the Society of Thoracic Surgeons suggests that the decision about when to treat a mycotic aneurysm should be made on a case-by-case basis. ${ }^{\text {? }}$

The Japanese Circulation Society suggests that if a patient has a ruptured mycotic aneurysm, the neurosurgery should be performed first, followed by the valve surgery after 2 to 3 weeks. ${ }^{10}$ No randomized, prospective studies have provided evidence as to the optimal timing of these operations. The strategy should be decided according to the pathophysiologic condition of each patient.

In our case, the interval was 3 days, which is the shortest interval reported to date. Because the hemorrhagic lesion increased in size, and the brain angiogram confirmed the mycotic aneurysm rupture, the decision was made to perform the neurosurgery first and the cardiac surgery second. We ordered a brain MRI 2 days after the craniotomy, to confirm that no brain hemorrhagic complications had occurred. The craniotomy wound healed well. After another multidisciplinary discussion, we decided to perform cardiac surgery 3 days after the neurosurgery, to minimize the risk of another brain stroke due to the large, mobile vegetations on the patient's mitral valve.

\section{CONCLUSIONS}

Open repair of the heart for IE patients with cerebrovascular complications can be performed safely as early as 3 days after craniotomy, with the aid of close collaboration between cardiac surgeons and neurosurgeons.

The authors gratefully acknowledge the help of Shadia Constantine, MD, FACP, MPH, for the excellent Englishlanguage editing of this article, and Fumiyuki Okamoto, MD, $\mathrm{PhD}$, for the supervision of the Department of Cardiovascular Surgery, Teine Keijinkai Hospital.

\section{References}

1. Eishi K, Kawazoe K, Kuriyama Y, Kitoh Y, Kawashima Y, Omae T. Surgical management of infective endocarditis associated with cerebral complications. Multicenter retrospective study in Japan. J Thorac Cardiovasc Surg. 1995;110:1745-55.

2. Kin H, Yoshioka K, Kawazoe K, Mukaida M, Kamada T, Mitsunaga Y, et al Management of infectious endocarditis with mycotic aneurysm evaluated by brain magnetic resonance imaging. Eur J Cardiothorac Surg. 2013;44:924-30.

3. Sakamoto T, Kano H, Miyahara S, Inoue T, Izawa N, Gotake Y, et al. Efficacy of nafamostat mesilate as anticoagulation during cardiopulmonary bypass for early surgery in patients with active infective endocarditis complicated by stroke. J Heart Valve Dis. 2014;23:744-51.

4. Asai T, Usui A, Miyachi S, Ueda Y. Endovascular treatment for intracranial mycotic aneurysms prior to cardiac surgery. Eur J Cardiothorac Surg. 2002;21:948-50.

5. Gillinov AM, Shah RV, Curtis WE, Stuart RS, Cameron DE, Baumgartner WA, et al. Valve replacement in patients with endocarditis and acute neurologic deficit. Ann Thorac Surg. 1996;61:1125-9; discussion 1130.

6. Fukuda W, Daitoku K, Minakawa M, Fukui K, Suzuki Y, Fukuda I. Infective endocarditis with cerebrovascular complications: timing of surgical intervention. Interact Cardiovasc Thorac Surg. 2012;14:26-30.

7. Fujii Y, Fukuda I, Kigawasa I, Yamabuki K, Noguchi Y. Mitral valve replacement secondary to resection of mycotic cerebral aneurysm in acute phase of bacterial endocarditis—a case report. Nihon Kyobu Geka Gakkai Zasshi. 1994;42:1231-4.

8. Kuki S, Yoshida K, Suzuki K, Matsumura R, Okuda A. Successful surgical management for multiple cerebral mycotic aneurysms involving both carotid and vertebrobasilar systems in active infective endocarditis. Eur J Cardiothorac Surg. 1994;8:508-10.

9. Byrne JG, Rezai K, Sanchez JA, Bernstein RA, Okum E, Leacche M, et al Surgical management of endocarditis: the Society of Thoracic Surgeons clinical practice guideline. Ann Thorac Surg. 2011;91:2012-9.

10. Niwa K, Nakazawa M, Miyatake K, Tateno S, Yoshinaga M, Japanese Circulation Society (JCS) Joint Working Groups for Guidelines for Management of Infective Endocarditis; Japanese Society of Pediatric Cardiology and Cardiac Surgery Joint Working Groups for Guidelines for Prophylaxis, Diagnosis and Management of Infective Endocarditis in Patients with Congenital Heart Disease. Survey of prophylaxis and management of infective endocarditis in patients with congenital heart disease: Japanese nationwide survey. Circ J. 2003;67:585-91. 